\title{
Prediction of psychosocial risks in teachers using data mining
}

\section{Autores}

Amelec Viloria, Jorge Rodríguez López, Nataly Orellano Llinás, Carlos Vargas Mercado, Luz Estela León Coronado, Ana María Negrete Sepulveda, Omar Bonerge Pineda Lezama

\begin{abstract}
Integrated management systems aim to improve these everyday situations that are inherent to work and cause for concern. In search for continuous improvement, it is necessary to innovate with techniques in areas that are not yet explored and that contribute to strategic decisionmaking processes, such as machine learning techniques or machine learning. In occupational safety and health management systems, it is important to carry out the proper follow-ups and process controls in any type of industry and organization whose relationship is direct. This paper presents the application of three methods related to data mining: Support Vector Machine algorithms, Naïve Bayes, and Genetic Algorithms to identify the degree of psychosocial risk in university teachers of the Mumbai University in India. The use of SVM easily recognizes physiological variables and the best prediction performance was achieved with $96.34 \%$ accuracy efficiency.

\section{Palabras clave}

Support vector machine, Naïve bayes, Genetic algorithms
\end{abstract}

